Erratum

\title{
Erratum to "Integer Codes Correcting Burst and Random Asymmetric Errors within a Byte" [J. Franklin Inst. 355 (2018) 981-996]
}

\author{
Aleksandar Radonjic*, Vladimir Vujicic \\ Institute of Technical Sciences of the Serbian Academy of Sciences and Arts, Knez Mihailova 35/IV, 11000 \\ Belgrade, Serbia \\ Available online 23 May 2018
}

An equation appearing on page 992 of the article is incorrect. The incorrect equation appearing thus:

$$
G=\frac{C_{R}}{T_{\text {total }} / K}=\frac{\left(3.5 \cdot 10^{9}\right) \cdot 128 \cdot K}{9 \cdot k+29 \cdot n_{T L}+4}
$$

Should in fact appear thus:

$$
G=\frac{C_{R}}{T_{\text {total }} / K}=\frac{\left(3.5 \cdot 10^{9}\right) \cdot 128 \cdot k}{9 \cdot k+29 \cdot n_{T L}+4}
$$

Also, a sentence appearing on page 922 is incorrect. The following statement:

one code with code rate 0.9922 has theoretical throughputs above $32 \mathrm{Gbps}$. Thus, it could

be candidates for use in ONWOAs operating at $32 \mathrm{Gbps}$ (e.g. 32G Fibre Channel network).

Should read thus:

...one code with code rate 0.9922 has theoretical throughput above 32 Gbps. Thus, it could be candidate for use in ONWOAs operating at 32 Gbps (e.g. 32G Fibre Channel network).

DOI of original article: 10.1016/j.jfranklin.2018.04.034

* Corresponding author.

E-mail addresses: sasa_radonjic@yahoo.com (A. Radonjic), vujicicv@uns.ac.rs (V. Vujicic). 